\title{
Selective criteria for the microbiological examination of faecal specimens
}

\author{
R A Bowman, J M Bowman, S A Arrow, T V Riley
}

\begin{abstract}
To assess the effectiveness of predetermined investigation criteria for the examination of faecal samples from inpatients, cultured stool specimens were prospectively examined for Salmonella spp, Shigella spp, Campylobacter spp and Clostridium difficile, and screened microscopically for intestinal parasites. Out of a total of 505 specimens, $421(83 \%)$ fulfilled the criteria for examination for $C$ difficile, 254 (50\%) for Salmonella spp, Shigella spp, and Campylobacter spp, and $87(17 \%)$ for intestinal parasites. Isolation rates for these organisms in those groups of patients where examination was indicated were $22.5 \%$ for $C$ difficile and $9 \cdot 1 \%$ for Salmonella spp, Shigella spp, and Campylobacter spp; the detection rate for parasites was $3 \cdot 5 \%$. In those patients where the criteria did not suggest investigation, the isolation or detection rates were $3.6 \%$ for $C$ difficile, $0 \%$ for Salmonella spp, Shigella spp, and Campylobacter spp, and $1.7 \%$ for intestinal parasites, suggesting that the use of predetermined investigation criteria was effective.
\end{abstract}

Diarrhoea is common in inpatients and laboratory investigation is often undertaken in an attempt to identify an aetiological agent. Both the requesting physician and the laboratory are faced with an ever increasing list of potential enteric pathogens, and most laboratories would not contemplate investigation for all these organisms. The decision as to which enteric pathogens should be sought is often made after many factors have been considered. These include macroscopic appearance of the stool; presence of leucocytes and erythrocytes on direct microscopic examination; clinical and travel history of the patient; and a history of antibiotics. Despite the use of such criteria, many laboratories still persist with "routine" stool cultures to exclude Salmonella spp, Shigella spp and Campylobacter spp. Historical considerations have undoubtedly played an important part in the perpetuation of this practice. Indeed, clinical and laboratory texts generally support this approach. ${ }^{12}$ Recent increases in the cost of health care have highlighted the need for laboratories to review protocols and provide cost effective services. With these considerations in mind we conducted a prospective trial to assess the effectiveness of various criteria for investigation for a range of enteric pathogens.

Methods

All patients studied were either inpatients or outpatients at the Sir Charles Gairdner Hospital, a 700 bed general hospital, between May and October 1990. During the six month period, the first stool sample received in the laboratory from each patient was cultured routinely for Salmonella spp, Shigella spp, Campylobacter spp and $C$ difficile by previously described methods ${ }^{3}$ and examined for enteric parasites using a formal-ethyl acetate concentration method. ${ }^{4}$ Repeat specimens were examined for these and other enteric pathogens depending on the macroscopic and microscopic appearance of the stool and the clinical history provided, or on request, by the attending physician. Direct microscopy was performed routinely on all specimens but examination for virus was not performed.

\section{INVESTIGATION CRITERIA}

Enteric pathogens were classified into three groups: group 1, $C$ difficile; group 2, Salmonella spp, Shigella spp and Campylobacter spp; and group 3, enteric parasites. Patient specimens were assessed as to whether investigation for any, or all, of these groups of organisms was warranted according to the clinical history provided on the request form, macroscopic appearance of the stool, and results of direct microscopic examination.

One or more of the following criteria for each group needed to be fulfilled

Group 1: (i) hospital stay of four days or more; (ii) loose or watery stool; (iii) erythrocytes or leucocytes seen on direct microscopy; (iv) history of exposure to antibiotics or cytotoxics.

Group 2: (i) hospital stay of less than four days; (ii) loose or watery stool; (iii) erythrocytes or leucocytes seen on direct microscopy.

Group 3: (i) presence of eosinophilia; (ii) history of chronic/persistent/recurrent diarrhoea; (iii) history of travel through areas in which parasitic infections were known to be endemic.

\section{Results}

A total of 505 stool specimens were examined for the complete range of enteric pathogens. There were no additional enteric pathogens found on repeat examinations.

Of 505 patients examined, 421 (83\%) fulfilled one or more of the criteria for culturing for $C$ difficile. Ninety five $(22 \cdot 5 \%)$ of these yielded $C$ difficile. Of the $84(17 \%)$ patients not fulfilling any of the criteria, three (3.6\%) yielded $C$ difficile.

Two hundred and fifty four patients (50\%) fulfilled one or more of the criteria outlined for culturing for Salmonella spp, Shigella spp and Campylobacter spp, and $23(9 \cdot 1 \%)$ of these yielded one of these organisms (20 Campylo- 
bacter spp, two Salmonella spp, and one Shigella spp). These organisms were not isolated from the remaining 251 patients who did not fulfil any of the criteria.

Of the 505 patients, $87(17 \%)$ met one or more of the relevant criteria for investigation for enteric parasites. Of these 87 patients, three (3.5\%) yielded intestinal parasites (one Giardia lamblia, one Enterobius vermicularis, and one case of concomitant Trichuris trichiura and Ancylostoma duodenale). Of the remaining 418 patients $(83 \%)$, seven $(1 \cdot 7 \%)$ were shown to harbour intestinal parasites (five Blastocystis hominis and two $G$ lamblia). Six of these were detected on direct microscopy and, therefore, would have been detected by routine examination. The remaining patient did not fulfill any of the criteria and a parasite ( $B$ hominis) was identified only after concentration.

\section{Discussion}

This study has clearly shown the higher isolation rate for $C$ difficile in a hospital population compared with Salmonella spp, Shigella spp, and, to a lesser extent, Campylobacter spp. Our previous study ${ }^{5}$ suggested that routine culture for $C$ difficile was not warranted provided an adequate clinical history was provided. We also questioned the need for routinely culturing all stool specimens for Salmonella spp and Shigella spp. The present study was designed to reappraise criteria for the examination for $C$ difficile and, at the same time, evaluate the adequacy of criteria for the examination for Salmonella spp, Shigella spp, Campylobacter spp and intestinal parasites. Therefore, the range of enteric pathogens considered in this study was representative of organisms commonly encountered in nosocomial and community acquired diarrhoeal illness (excluding virus).

Using the criteria described, $83 \%$ of stools required examination for $C$ difficile, more than the $57 \%$ in our previous study. ${ }^{5}$ This presumably reflects the additional requirement to look for $C$ difficile in those patients who had been in hospital for four or more days. In those patients where culture for $C$ difficile was indicated, the isolation rate was $22.5 \%$, also higher than in our previous study, ${ }^{5}$ and further highlighting the major part played by $C$ difficile in nosocomial diarrhoea within the hospital environment. $^{6-9}$ It may be that the incidence of diarrhoea due to $C$ difficile is increasing or that greater awareness of the problem is prompting the investigation of individuals at risk. In the 84 patients where culture for $C$ difficile was not indicated, three $(3.6 \%)$ yielded $C$ difficile. Because of these findings and because our investigation criteria indicated that $83 \%$ of samples should be investigated for $C$ difficile, we now suggest that the routine examination of all faecal specimens for $C$ difficile may be warranted.

Two hundred and fifty four of the 505 patients $(50 \%)$ fulfilled one or more of the criteria for culturing for Salmonella spp, Shigella spp, and Campylobacter spp. These organisms were isolated only in this group of patients. A more detailed examination of the patients that yielded Salmonella spp, Shigella spp, or Campylobacter spp showed that all had been in hospital for less than four days. Accordingly, we suggest that stools from patients who have been in hospital for more than three days do not need culturing for Salmonella spp, Shigella spp, and Campylobacter spp. Our findings strongly support the view of Siegel et al, who, in a three year retrospective study, showed that out of a total 281 positive results there was one infection only from this group of pathogens (a Salmonella spp) in a patient who had a hospital stay in excess of three days. ${ }^{10}$

The issue of examination for intestinal parasites was less elear. The criteria we used appeared less effective as seven $(1 \cdot 7 \%)$ patients shown to harbour intestinal parasites did not fulfill any of the criteria. However, parasites in six of these patients were detected by direct microscopic examination which we use routinely to detect faecal erythrocytes and leucocytes. Therefore, only one of $505(0.2 \%)$ patients would have been misdiagnosed. The need for investigation for intestinal parasites remains principally a clinical decision.

Ten years ago our laboratory routinely investigated all stool samples for Salmonella spp, Shigella spp, and intestinal parasites. Culture for $C$ difficile was only undertaken when indicated by the criteria described previously. ${ }^{5}$ With the emergence of $C$ difficile as almost the sole cause of nosocomially acquired diarrhoea, we have had to review our approach. We now examine all stool specimens for $C$ difficile. We culture for Salmonella spp, Shigella spp and Campylobacter spp when the patient has been in hospital for less than four days, and only examine for enteric parasites when specifically requested by the ordering physician. Examination for other enteric bacterial pathogens is also only undertaken when specifically indicated. This is a cost effective approach which has not reduced the quality of patient care, and is supported by similar studies. ${ }^{710}$

1 Guerrant RL. Principles and syndromes of enteric infection. In: Mandell GL, Douglas RG, Bennett JE, eds. Principles and practices of infectious diseases. 3rd edn. New York: Churchill Livingstone, 1990:837-50.

2 Sack RB, Tilton RC, Weissfield AS. Laboratory diagnosis of bacterial diarrhoea (Cumitech 12). Washington, DC: American Society for Microbiology 1980:5.

3 Riley TV, Bowman RA, Carroll SM. Diarrhoea associated with Clostridium difficile in a hospital population. Med 7 Aust 1983;i:166-9.

4 Smith JW, Bartlett MS. Diagnostic parasitology: Introduction and methods. In: Lennette EH, Balaws A, Hausler tion and methods. In: Lennette EH, Balaws A, Hausler WJ, Shadomy HJ, eds. Manual of clinical microbiology, 4th edn. Washingto

5 Bowman RA, Riley TV. Routine culturing for Clostridium difficile? Pathology 1984;16:240-2.

6 Thibault A, Miller MA, Gaese C. Risk factors for the development of Clostridium difficile associated diarrhoea during a hospital outbreak. Infect Control Hosp Epidemiol 1991;12:345-8.

7 Yanelli B, Gurevich I, Schoch PE, Cunha BA. Yield of stool cultures, ova and parasite tests, and Clostridium difficile determinations in nosocomial diarrhoeas. Am $\mathcal{f}$ Infect Cont 1988;16:246-9.

8 Johnson S, Gerding DN, Olson MM, et al. Prospective, controlled study of vinyl glove use to interrupt Clostridium difficile nosocomial transmission. Am $7 \mathrm{Med}$ 1990;88:137-40.

9 Eriksson S, Aronsson B. Medical implications of nosocomial infection with Clostridium difficile. Scand $\mathcal{F}$ Infect Dis infection with

10 Siegel DL, Edelstein PH, Nachamkin I. Inappropriate testing for diarrheal diseases in the hospital. $\mathscr{f} A M A$ 1990; 16:979-82. 